\title{
The Determination of the Parameters of a Vibration Machinef the Internal Compaction of Concrete Mixtures
}

\author{
Alexander Maslov ${ }^{1}$, Janar Batsaikhan ${ }^{2}$, Ruslan Puzyr $^{1}$, Yulia Salenko ${ }^{1}$ \\ ${ }^{1}$ Kremenchuk Mykhailo Ostrohradskyi National University \\ ${ }^{2}$ Research and production center $« M C P C g r »$ \\ *Corresponding author E-mail: kmto.43@gmail.com
}

\begin{abstract}
The determination of the rational parameters of a vibration machine for the internal compaction of concrete mixtures. We researched the "vibration machine - concrete medium" system wherein the concrete medium is represented by a system with distributed parameters that takes into account the resilient, viscous, inertial and energy properties of the compacted concrete medium. We researched the propagation of the viscous-resilient-plastic deformation waves in the contracted concrete mixture represented in the form of a half-space. We determined the amplitudes of the oscillations and stresses occurring in the compacted mixture depending on its physical and mechanical characteristics. We determined the oscillation amplitude of the vibration machine, identified its rational parameters. We determined the regularity of the motion of the compacted mixture and the vibration machine depending on the physical and mathematical characteristics of the compacted mixture, the thickness and width of the compacted layer, the angular oscillation frequency and the amplitude of the exciting force. The presented dependences enable the substantiation of the rational parameters of the vibration machine and the modes of vibratory action.
\end{abstract}

Keywords: amplitude; concrete mixture; internal compaction; parameters; stresses; vibration machine.

\section{Introduction}

Topicality Of The Paper. The internal (deep) vibrating of the concrete mixtures is energetically most profitable of all the compaction methods, as a vibratory working body submerged in the compacted medium directly transfers the vibratory action to it with minimal energy losses. For this purpose, immersion vibrators $[1-4]$ with the diameter of the head (cap) from 36 to $76 \mathrm{~mm}$ are used. For the technological equipment hinged immersion vibrators with the cap diameter from 75 to $133 \mathrm{~mm}$ are used. These vibration machines provide the processing and compaction of the concrete mixture in the radius from 200 to $300 \mathrm{~mm}$ depending on the cap diameters and the consistence of the concrete mixture. For the acceleration and complex mechanization of packing and compacting of concrete mixtures one uses packages of immersion vibrators with individual drives for each of them mounted in the form of working equipment on cranes or small-sized tractors by means of special suspension brackets [5]. The design of these structures is very complicated and they can only be used for a big amount of concrete casting.

To increase the active zone of vibration, we designed a planar immersion compactor [6] made in the form of a vertical flat plate equipped with two immersion vibrators with an individual drive of each of them. This immersion compactor cannot be used in construction as a manual mechanism due to its big weight. The efficiency of the use of the planar immerse vibrator was not researched so it was not widely used.

It should be mentioned that immersion compactors equipped with planetary vibrators [7] quickly fail during long-term operation.
Therefore creation of a high-technology vibration machine equipped with a flat working body, characterized by a simple design, high reliability and providing immersion (deep) compaction of concrete mixtures of different consistence is a topical problem.

For the efficient operation of the vibration machine it is necessary to thoroughly choose its basic parameters and modes of vibratory action on the compacted medium, represented by a rheological model in the form of a resilient Hooke's model, Newton's viscous body, the Kelvin-Vogt or Maxwell resilient-viscous body [8 - 9]. Sufficiently accurate results can be obtained if the compacted medium is presented in the form of continuum [10 - 12], and its oscillations are described by a wave equation [13 - 14], taking into account the resilient and viscous properties of the compacted material. The use of these methods does not take into consideration the inertial properties and friction of the concrete mixture components at their reorientation, approaching, deformation. That is why to choose the basic parameters of the vibration machine and the modes of the internal vibratory action on the concrete mixture, a rheological model for the distributedparameter continuum should be used, taking into account the resilient and non-resilient resistance forces as well as resistance caused by the action of the internal friction and the inertia of the concrete mixture mineral components.

The purpose of this research is determination of the rational parameters of the vibration machine for the internal compaction of concrete mixtures based on the analysis of its interaction with the compacted concrete mixture represented by a half-space in the form of a system with distributed parameters and the identification of the rational modes of the vibratory action on the concrete mixture by a flat working body. 


\section{The Materials and Results of the Study}

Nowadays, immersion vibrators are indispensable during the manufacture of structures from mass concrete as well as during concrete blocks production. With the objective of the reduction of energy consumption, the simplification of the design and the increase of the vibration active zone we worked out an immersion compactor with a flat vertical plate equipped with a vibratory exciter of horizontal oscillations.

Essentially, the regularity of the oscillations of the immersion compactor used for compacting and processing concrete mixtures is determined by the physical and mechanical characteristics of the concrete mixture, the directions and modes of the vibratory action, the thickness of the concrete layer subjected to the vibratory action and the basic parameters of the vibratory machine. As a result of the vibration compactor vibratory actions, inertial, resilient and non-resilient resistance forces occur in the concrete mixtureessentially influencing the compaction process. The character of the occurring resistance forces is determined by the frequency and amplitude of oscillations, the consistence and granulometric composition of the mixture, the thickness of the compacted layer, the direction and the type of the vibratory action on the compacted medium. A sufficiently accurate determination of the forces of interaction of the vibratory immersion compactor with the processed medium creates prerequisites for the identification of the vibration machine rational parameters enabling the efficient compaction and processing of the concrete mixtures of low energy consumption and high productivity.

To determine the character of the vibration machine working body interaction with the concrete mixture we will research the dynamic system shown in Fig.1. Here a vibration machine for the immersion compaction of concrete mixtures consists of flat vertical plate 1 , the upper part of which is equipped with oscillation vibratory exciter 2 . Vertical plate 1 is submerged into concrete mixture 3, represented by a system with distributed parameters. The concrete mixture is put on base 4 . The considered design diagram assumes that under the action of oscillation vibratory exciter 2 vertical plate 1 performs only horizontal oscillations perpendicular to plate 1 plane. In this case under the action of the horizontal harmonic force $Q \sin \omega t$ the immersion vibratory compactor oscillates horizontally and performs a vibratory action on the compacted mixture. Under this action the mixture transfers into the thixotropic condition, the air is removed out of it and it becomes more compact. Here $Q$ - the amplitude of the horizontal exciting force; $\omega-$ the angular frequency of the forced oscillations; $t$ - time. In this case the concrete mixture is represented by a half-space in relation to each side of the vertical plate.

The compacted medium rheological model taking into account the action of resilient, dissipative and inertial forces occurring in this medium under the dynamic action can be represented in the form of a scheme shown in Fig. 2. In accordance with the adopted rheological model, we research the one-axis stressed state occurring in the compacted medium only in horizontal direction under the action of the vibratory excitation $Q \sin \omega t$ that can be represented in the form of an imaginary part of a complex function, i.e. $Q \sin \omega t=I_{m}\left(Q e^{i \omega t}\right)$. In the future sign $I_{m}$ will be neglected for convenience. We will represent the dependence between the stress and deformation in the concrete medium in the form of an equation taking into account the action of the resilient, dissipative and inertial forces essentially influencing the occurrence of stresses:

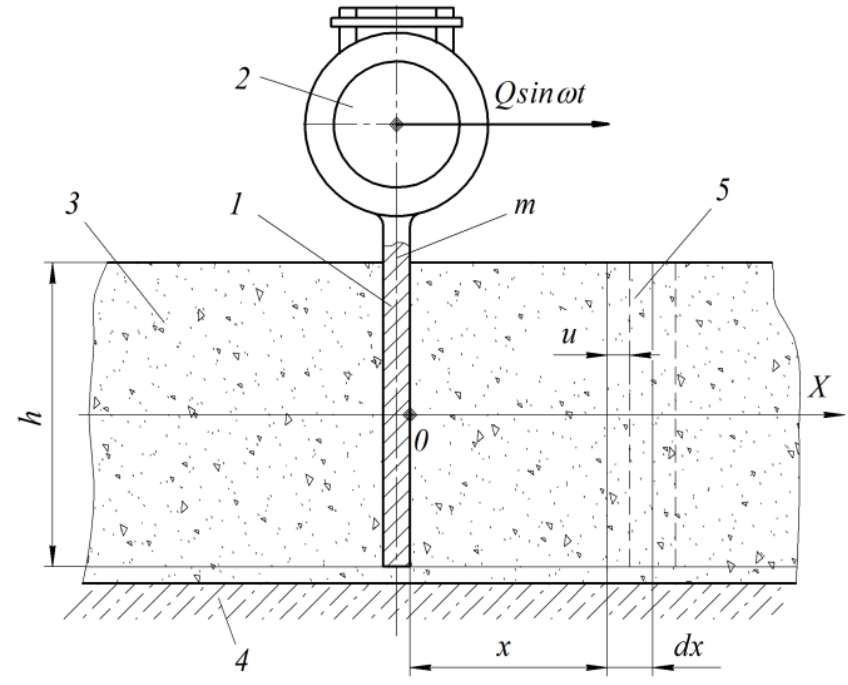

Fig. 1: The design diagram of the planar immerse compactor

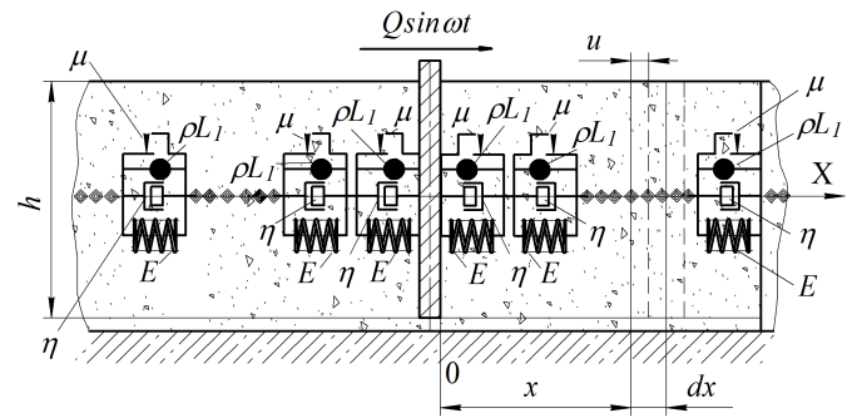

Fig. 2: The rheological model of compacted mixture

$\sigma(x, t)=E \frac{\partial u(x, t)}{\partial x}+\eta \frac{\partial u(x, t)}{\partial t}-\rho L_{1} \frac{\partial^{2} u(x, t)}{\partial t^{2}}+\mu u(x, t)$,

where $\sigma(x, t)$ - stresses occurring in the compacted layer in the direction of the coordinate axis $X ; u$ and $x$ - Eulerian and Lagrangian coordinates (Fig. 1, pos. 5); $E$ - the dynamic module of the resilient deformation of the mixture concrete layer; $\eta-$ the dynamic viscosity coefficient taking into account the internal friction in the concrete mixture; $\rho$ - the concrete mixture density; $L_{1}$ - the reduced effective length of the concrete mixture compacted layer in the direction of the coordinate axis $X ; \mu-$ the resistance coefficient taking into account the energy consumed by the destruction of the internal links, air displacement, particles reorientation and other compacted medium phenomena accompanying the vibratory compaction .

Based on expression (1) we describe the concrete mixture layer oscillations in the direction of coordinate $X$ in time $t$ by the following wave equation of oscillations:

$$
\begin{aligned}
& \frac{\partial}{\partial x}\left[E \frac{\partial u(x, t)}{\partial x}+\eta \frac{\partial u(x, t)}{\partial t}-\rho L_{1} \frac{\partial^{2} u(x, t)}{\partial t^{2}}+\mu u(x, t)\right]= \\
& =\rho \frac{\partial^{2} u(x, t)}{\partial t^{2}} .
\end{aligned}
$$

The solution to equation (2) can be represented in the form of the imaginary part of function [13]:

$u(x, t)=U(x) e^{i \omega t}$, 
where $U(x)$-the oscillations complex amplitude that is to meet the edge (boundary) conditions of the design diagram shown in Fig. 1.

The behavior of the considered dynamic system will depend on the boundary conditions. As a result of the motion of the planar vibration compactor directly along coordinate $X$ from the zero position, the front wall of the planar compactor with coordinate $x=0$ performs the concrete mixture compaction, and the reverse wall moving in the same direction will perform a stretching action on the compacted medium. As it is considered [14] that the physical and mechanical characteristics of the concrete mixture at vibratory action are equal at both compression and stretching, it is sufficient to analyze the interaction of the surface compactor only on one side, respectively doubling the area of interaction of the concrete mixture with the surface compactor. In this case the boundary conditions will have the form:

$$
\begin{aligned}
& F \frac{\partial u(0, t)}{\partial x}+\eta F \frac{\partial u(0, t)}{\partial t}-\rho L_{1} F \frac{\partial^{2} u(0, t)}{\partial t^{2}}+\mu F u(0, t)= \\
& =m \frac{\partial^{2} u(0, t)}{\partial t^{2}}+Q e^{i \omega t} ; \\
& \frac{\partial u(L, t)}{\partial x}=0,
\end{aligned}
$$

where $m$ - the mass of the planar immersion vibratory compactor; $F$ - the area of the vertical plate contacting with the compacted concrete mixture; $L$ the length of a half-wave of the vibration excitation distributed in the concrete medium,

$$
L=\frac{\pi}{\omega} \sqrt{\frac{E}{\rho}} .
$$

The first boundary condition (5) describes the interaction of the vertical plate with the compacted concrete mixture and the second one (6) shows that excitation wave distribution is not limited by anything and at the distance equal to a quarter of the wave the relative deformation of the compacted medium equals to zero, i.e. $\frac{\partial u(L, t)}{\partial x}=0$. Substituting function (3) into wave equation (2), we get an equation for the determination of the oscillations complex amplitude:

$$
\frac{\partial^{2} U(x)}{\partial x^{2}}+\frac{\mu+\rho L_{1} \omega^{2}+i \eta \omega}{E} \cdot \frac{\partial U(x)}{\partial x}+\frac{\rho \omega^{2}}{E} U(x)=0 ;
$$

For equation (7) we formulate a characteristic equation and find its roots in the following way:

$$
k_{1-2}=-(\delta+i \xi) \pm i \sqrt{\frac{\rho \omega^{2}}{E}+\xi^{2}-\delta^{2}-2 i \cdot \delta \xi},
$$

where $\delta$ - the coefficient of excitation attenuation in the compacted layer of the concrete mixture; $\xi$ - the coefficient of dissipation,

$$
\begin{aligned}
& \delta=\frac{\mu+\rho L_{1} \omega^{2}}{2 E} ; \\
& \xi=\frac{\eta \omega}{2 E} .
\end{aligned}
$$

Using roots (8) of the characteristic equation we find the solution to equation (7) in the following form:

$U(x)=\left(B_{1} e^{-i \tilde{k} x}+B_{2} e^{i \tilde{k} x}\right) \exp [-(\delta+i \xi) x]$,

where $B_{1}$ and $B_{2}$ - integration constants (complex functions), determined from the boundary conditions (4) and (5); $\tilde{k}-$ the complex wave number,

$\tilde{k}=\sqrt{\chi-2 i \delta \zeta}$

$\chi=\frac{\rho \omega^{2}}{E}+\xi^{2}-\delta^{2}$

Based on the obtained dependence (12) the complex wave number $\tilde{k}$ can be represented in the following form [5]:

$\tilde{k}=k-i \alpha$,

where $k$ - the wave number; $\alpha$ - the absorption coefficient characterizing the reduction of the excitation amplitude during moving away from the source of vibratory action.

To determine wave number $k$ and absorption coefficient $\alpha$ we equate expressions (12) and (14), square the right and the left parts of the obtained equality and separating the imaginary and the real parts we obtain a system of equations

$\left\{\begin{array}{l}k^{2}-\alpha^{2}=\chi \\ \alpha k=\delta \xi\end{array}\right.$

wherefrom we find

$k=\sqrt{0,5 \chi+\sqrt{0,25 \chi^{2}+\xi^{2} \delta^{2}}} ;$

$\alpha=\sqrt{-0,5 \chi+\sqrt{0,25 \chi^{2}+\xi^{2} \delta^{2}}}$.

Using expressions (11) and (14), we represent the solution to equation (6) in the following form:

$U(x)=\left\{B_{1} \exp [-(i k+\alpha) x]+B_{2} \exp [(i k+\alpha) x]\right\} \times$
$\times \exp [-(\delta+i \xi) x]$

Substituting dependence (18) into complex function (3), we find the solution to equation (2) in the following complex form:

$$
\begin{aligned}
& u(x, t)=e^{-\delta x}\left\{B_{1} \exp [-(i k+\alpha) x]+B_{2} \exp [(i k+\alpha) x]\right\} \times \\
& \times \exp [i(\omega t-\xi x)] .
\end{aligned}
$$

Substituting dependence (19) into boundary condition (5), we find the relation between integration constants $B_{2}$ and $B_{1}$ :

$$
B_{2}=-B_{1} \frac{M-i N}{d+i \lambda},
$$

where

$$
\begin{aligned}
& M=e^{-\alpha L}[(\delta+\alpha) \cos k L+(k+\xi) \sin k L] ; \\
& N=e^{-\alpha L}[(\delta+\alpha) \sin k L-(k+\xi) \cos k L] ;
\end{aligned}
$$


$d=e^{\alpha L}[(\delta-\alpha) \cos k L+(k-\xi) \sin k L]$

$\lambda=e^{\alpha L}[(\delta-\alpha) \sin k L-(k-\xi) \cos k L] ;$

Substituting the relation between complex amplitudes (20) into expression (19), we find the solution to equation (3) in the following form:

$$
u(x, t)=B_{1} e^{-\delta x}\left[\exp [-(i k+\alpha) x]-\frac{(M-i N) \exp [(i k+\alpha) x]}{d+i \lambda}\right] \times
$$$$
\times \exp [i(\omega t-\xi x)] \text {. }
$$

By substituting dependence (25) into boundary condition (4) we find integration constant $B_{1}$ (a complex function):

$$
\begin{aligned}
& B_{1}=\frac{Q(d+i \lambda)}{(d-M)+i(\lambda+N)}\left[E F(\alpha+i k) \frac{(d+M)+i(\lambda-N}{(d-M)+i(\lambda+N)}-\right. \\
& \left.-m \omega^{2}-0,5 F\left(\rho L_{1} \omega^{2}+\mu+i \cdot 0,5 \eta \omega\right)\right]^{-1} .
\end{aligned}
$$

Substituting integration constant $B_{1}$ (26) into dependence (19), we find the solution to oscillation wave equation (2), meeting boundary conditions (4) and (5), in the complex form:

$$
\begin{aligned}
& u(x, t)=Q \frac{(d+i \lambda) \exp [-(i k+\alpha) x]-(M-i N) \exp [(i k+\alpha) x]}{(d-M)+i(\lambda+N)} \times \\
& \times\left[-m \omega^{2}-0,5 F\left(\rho L_{1} \omega^{2}+\mu+i \cdot 0,5 \eta \omega\right)+\right. \\
& \left.+E F(\alpha+i k) \frac{(d+M)+i(\lambda-N)}{(d-M)+i(\lambda+N)}\right]^{-1} \exp [-\delta x+i(\omega t-\xi x)] .
\end{aligned}
$$

We multiply the numerator and denominator of expression $\frac{(d+M)+i(\lambda-N)}{(d-M)+i(\lambda+N)}$, being in brackets in dependence (27), by a complex function conjugate to denominator, i.e. by $(d-M)-i(\lambda+N)$. Then, transforming the obtained expression we get

$$
\begin{aligned}
& u(x, t)=Q \frac{(d+i \lambda) \exp [-(i k+\alpha) x]-(M-i N) \exp [(i k+\alpha) x]}{(d-M)+i(\lambda+N)} \times \\
& \times \frac{1}{\left[c_{b}-\left(m+m_{b}\right) \omega^{2}\right]-i b_{b} \omega} \exp [-\delta x+i(\omega t-\xi x)],
\end{aligned}
$$

where $c_{b}-$ the reduced rigidity of the compacted concrete mixture

$c_{b}=E F \frac{\alpha\left(d^{2}+\lambda^{2}\right)+2 k(d N+\lambda M)}{(d-M)^{2}+(\lambda+N)^{2}} ;$

$m_{b}$ - the reduced mass of the compacted concrete mixture,

$$
m_{b}=E F \alpha \frac{M^{2}+N^{2}}{\left[(d-M)^{2}+(\lambda+N)^{2}\right] \omega^{2}}+0,5 F\left(\frac{\mu}{\omega^{2}}+\rho L_{1}\right) ;
$$

$b_{b}-$ the reduced coefficient of the non-resilient resistance of the compacted concrete mixture,

$$
b_{b}=E F \frac{2 \alpha(d N+\lambda M)-k\left(d^{2}+\lambda^{2}-M^{2}-N^{2}\right)+Z \omega \xi}{\left[(d-M)^{2}+(\lambda+N)^{2}\right] \omega} ;
$$

We multiply the numerator and denominator of expression (28) by complex functions $\left[c_{b}-\left(m+m_{b}\right) \omega^{2}\right]+i b_{b} \omega \quad$ and $(d-M)-i(\lambda+N)$, conjugate to the complex functions in the denominator and, separating from the obtained dependence the imaginary part of the formed complex function and transforming it, we obtain the desired solution to the wave equation (2), meeting the boundary conditions (4) and (5), in the following form:

$$
\begin{aligned}
& u(x, t)=\frac{Q}{\sqrt{(d-M)^{2}+(\lambda+N)^{2}} \sqrt{\left[c_{b}-\left(m+m_{b}\right) \omega^{2}\right]^{2}+b_{b}^{2} \omega^{2}}} \times \\
& \times\left\{\exp [-(\delta+\alpha) x] \sqrt{M^{2}+N^{2}} \cos \left[\omega t-(\xi-k) x-\varphi+\theta_{1}\right]+\right. \\
& \left.+\exp [-(\delta-\alpha) x] \sqrt{\lambda^{2}+d^{2}} \cos \left[\omega t-(\xi+k) x-\varphi-\theta_{2}\right]\right\}
\end{aligned}
$$

where $\varphi$ - phase shift between the amplitude of the exciting force and movement,

$\varphi=\varphi_{2}-\varphi_{1}$

$\varphi_{1}=\operatorname{arctg} \frac{b_{b} \omega}{c_{b}-\left(m+m_{b}\right) \omega^{2}}$

$\varphi_{2}=\operatorname{arctg} \frac{\lambda+N}{d-M}$

$\theta_{1}=\operatorname{arctg} \frac{M}{N}$

$\theta_{2}=\operatorname{arctg} \frac{d}{\lambda}$

The obtained solution (32) analytically describes the law of the oscillations of the compacted layer of the analyzed "vibration machine - concrete medium" dynamic system depending on coordinate $x$, i.e. at $L \leq x \geq 0$. At $x=0$ dependence (32) describes the law of oscillations of the mixture layer adjacent to the vertical plate and simultaneously the law of oscillations of the planar immersion compactor, i.e.

$u(0, t)=A \sin \left(\omega t+\varphi_{1}\right)$,

where $A-$ the amplitude of the oscillations of the vertically installed plate of the immersion compactor,

$$
A=\frac{Q}{\sqrt{\left[c_{b}-\left(m+m_{b}\right) \omega^{2}\right]^{2}+b_{b}^{2} \omega^{2}}} .
$$

Substituting dependence (32) into equation (1), we determine the alteration of stresses occurring in the compacted layer under the action of vibratory excitation $Q \sin \omega t$ :

$\sigma(x, t)=\frac{A E \sqrt{\left(M^{2}+N^{2}\right)\left[(k+\xi)^{2}+(\delta+\alpha)^{2}\right.}}{\sqrt{(d-M)^{2}+(\lambda+N)^{2}}} \times$

$\times \exp [-(\delta+\alpha) x] \cos \left[\omega t-(\xi-k) x-\varphi+\theta_{1}+\varphi_{3}\right]$;

$+\frac{A E \sqrt{\left(\lambda^{2}+d^{2}\right)\left[(k-\xi)^{2}+(\delta-\alpha)^{2}\right.}}{\sqrt{(d-M)^{2}+(\lambda+N)^{2}}} \times$

$\times \exp [-(\delta-\alpha) x] \cos \left[\omega t-(\xi+k) x-\varphi-\theta_{2}-\varphi_{4}\right]$; 
where $\varphi_{3}, \varphi_{4}-$ the phases shift angles,

$\varphi_{3}=\operatorname{arctg} \frac{\alpha}{k} ; \quad \varphi_{4}=\operatorname{arctg} \frac{\eta \omega}{\mu+\rho L_{1} \omega^{2}}$.

The stresses occurring in the layer of the concrete mixture where it contacts with the vertical plate will equal:

$\sigma(0, t)=\frac{A E \sqrt{\left(M^{2}+N^{2}\right)\left[(k+\xi)^{2}+(\delta+\alpha)^{2}\right.}}{\sqrt{(d-M)^{2}+(\lambda+N)^{2}}} \times$

$\times \cos \left(\omega t-\varphi+\varphi_{3}+\theta_{1}\right)+$

$+\frac{A E \sqrt{\left(\lambda^{2}+d^{2}\right)\left[(k-\xi)^{2}+(\delta-\alpha)^{2}\right.}}{\sqrt{(d-M)^{2}+(\lambda+N)^{2}}} \times$

$\times \cos \left(\omega t-\varphi-\varphi_{4}-\theta_{2}\right)$,

Using expressions $(29-31)$, we determine the specific values: -of the reduced rigidity $\left(c_{b y}\right)$ of the compacted concrete mixture,

$c_{b y}=\frac{c_{b}}{F}=E \frac{\alpha\left(d^{2}+\lambda^{2}\right)+2 k(d N+\lambda M)}{(d-M)^{2}+(\lambda+N)^{2}} ;$

-of the reduced mass $\left(m_{b y}\right)$ of the compacted concrete mixture,

$m_{b y}=\frac{m_{b}}{F}=E \alpha \frac{M^{2}+N^{2}}{\left[(d-M)^{2}+(\lambda+N)^{2}\right] \omega^{2}}+0,5\left(\frac{\mu}{\omega^{2}}+\rho L_{1}\right) ;$

-of the reduced coefficient of non-resilient resistance $\left(b_{b y}\right)$ of the compacted concrete mixture,

$b_{b y}=\frac{b_{b}}{F}=E\left[\frac{2 \alpha(d N+\lambda M)-k\left(d^{2}+\lambda^{2}-M^{2}-N^{2}\right)}{\left[(d-M)^{2}+(\lambda+N)^{2}\right] \omega}+\xi\right]$.

The obtained expressions $(43-45)$ can be used for the research of a continual-discrete calculation model, describing the interaction of the immersion compactor with the concrete mixture at various methods of vibratory actions, configuration of the compacted product and different values of the area of the surfaces of the interaction of the working body and the concrete medium.

Using expressions (43 - 45), the amplitude of stresses occurring in the compacted layer of concrete mixture depending on the action of resilient and inertial forces and forces of non-resilient resistance can be determined by the simplified formula with a sufficient degree of accuracy, i.e.

$\sigma(x)=A e^{-(\delta-\alpha) x}\left(m_{b y} \omega^{2}+\left|b_{b y}\right| \omega+E / L_{1}\right)$.

For the considered design diagram, the effective length of the compacted layer is assumed equal to a quarter of the length of the wave of the excitation propagated in the concrete medium:

$L_{1}=\frac{\pi}{2 \omega} \sqrt{\frac{E}{\rho}}$.

Figs. 3 - 5 contain the variation of the coefficient of the reduced rigidity, the reduced coefficient of non-resilient resistance and reduced mass of concrete mixtures of different consistence depending on the compacted medium relative density $\varepsilon$ found by the following dependence: $\varepsilon=\frac{\rho-\rho_{0}}{\rho_{k}-\rho_{0}}$,

where $\rho_{0}-$ the density of the concrete mixture in the uncompacted state (initial density); $\rho_{k}$ - the density of the concrete mixture in the compacted state; $\rho$ - the current value of density.

The data shown in Fig. $3-5$ are obtained with the use of theoretical dependences $(29-31)$ for the planar immersion compactor performing strictly horizontal oscillations in the direction of coordinate $x$, with the following basic parameters: the immersion compactor mass $-m=7 \mathrm{~kg}$; the amplitude of the exciting force of the oscillations vibratory exciter $-Q=0.981 \mathrm{kN}$; the angular frequency of the forced oscillation $-\omega=292 \mathrm{rad} / \mathrm{s}$; the area of the vertical plate surface interacting with the concrete mixture (at the two-sided contact) $-F=800 \mathrm{~cm}^{2}$; the amplitude of the vertical plate oscillations in a strictly horizontal direction, perpendicular to the plane of the interaction of the vertical plate with the concrete mixture at idle running (without interaction with the concrete mixture) $-A=0.167 \mathrm{~cm}$.

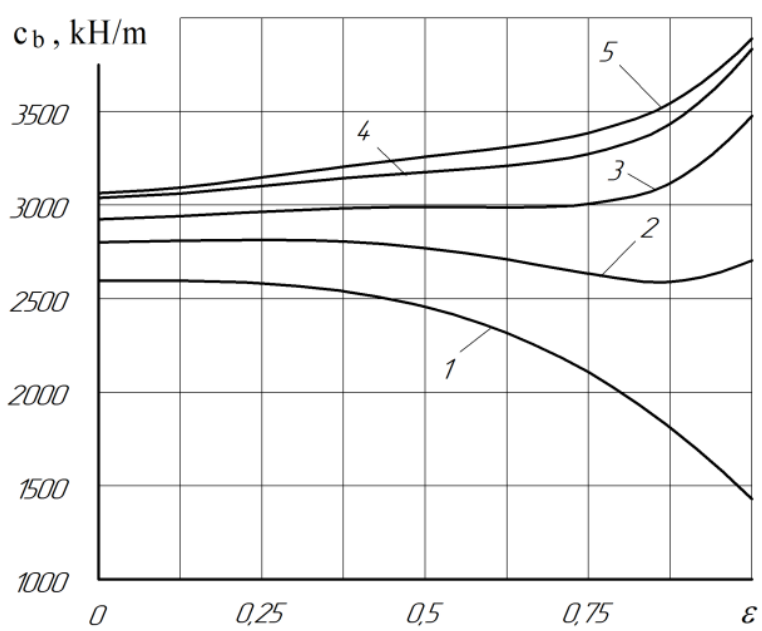

Fig. 3: The variation of the coefficient of the reduced rigidity of concrete mixtures $c_{b}$ of different consistence depending on their relative density $\varepsilon: 1$ - at mixture rigidity $S=6 \mathrm{~s} ; 2$ - at $S=30 \mathrm{~s} ; 3$ - at $S=60 \mathrm{~s} ; 4$ - at $\mathrm{S}=90 \mathrm{~s} ; 5-$ at $\mathrm{S}=120 \mathrm{sc}$

The analysis of the obtained dependences reveals that the characters of the values of the reduced coefficient of the compacted mixture rigidity are of different directions and depend on both relative density of the concrete mixture and its consistence (Fig. 3). With the increase of mobility and relative density for mixtures with $\mathrm{S}=6 \mathrm{~s}$ their reduced coefficient of rigidity decreases 


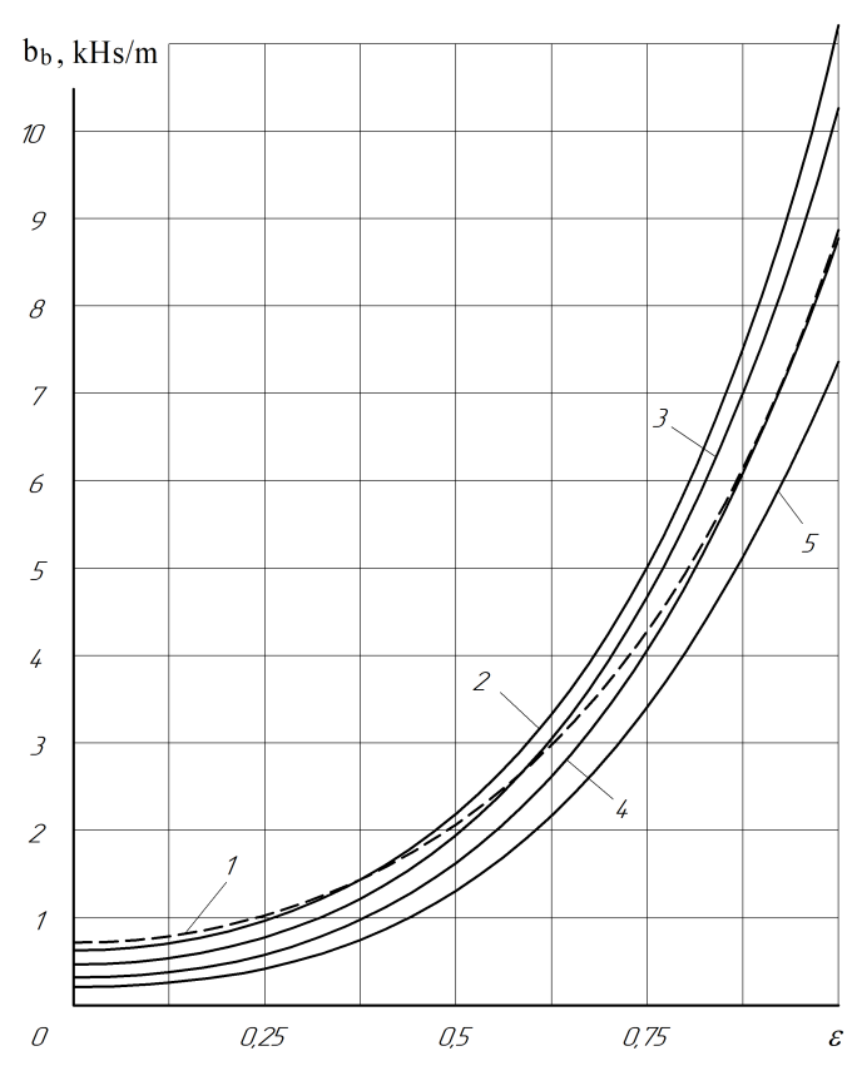

Fig. 4: The variation of the coefficient of the non-resilient of resistance $b_{b}$ of concrete mixtures of different consistence depending on their relative density $\varepsilon: 1-$ at rigid mixture $S=6 \mathrm{~s} ; 2-$ at $S=30 \mathrm{~s} ; 3-$ at $S=60 \mathrm{~s}$; $4-$ at $\mathrm{S}=90 \mathrm{~s} ; 5-$ at $\mathrm{S}=120 \mathrm{~s}$

It can be explained by the fact that at vibratory action on a halfspace, when the relative density of the compacted mixture increases, big masses of concrete mixture are involved into the oscillation process due to the growth of the length of the wave of the propagated excitation. It impedes the growth of the reduced coefficient of the processed medium rigidity in spite of the essential increase of the mixture resilient deformation dynamic module $E$. The reduced coefficient of the non-resilient of concrete mixtures resistance $b_{b}$ essentially depends on the relative density and also on their consistence.

Reduced mass $m_{b}$ insignificantly depends on the concrete mixture consistence but increases greatly in the process of compaction with the increase of the relative density of the mixture $\varepsilon$. Thus, the values of the reduced coefficients of rigidity, non-resilient resistance and mass determining the interaction of the planar immersion vibratory compactor with the concrete medium essentially depend on consistence, physical and mechanical characteristics and relative density of the concrete mixture, boundary conditions, the frequency of the vibratory action and the area of the interaction of the vertical plate with the compacted concrete medium. These parameters, along with the amplitude of the exciting force, determine the amplitude of the immersion compactor oscillation amplitude. After the introduction of the immersion compactor into the processed medium its oscillation amplitude, which equals $0.167 \mathrm{~cm}$ at idle running, decreases and, depending on the mixture consistence, makes $0.0672-0.1044 \mathrm{~cm}$ at the beginning of the compaction (Fig. 6). The immersion compactor has the largest oscillation amplitude when it interacts with the concrete mixture with the rigidity of 120 s.

When the density of the concrete mixture grows, the immersion compactor oscillation amplitude decreases and at the final stage of compaction it makes $0.0206-0.0346 \mathrm{sm}$, and at the increase of the concrete mixture rigidity the values of the oscillation amplitude are higher. It can be explained by the fact that when the concrete mixture density grows, its reduced mass involved into the oscillation process grows, and the dissipative forces providing energy absorption also grow. The increase of stresses, shown in Fig 7, occurring in the concrete mixture at its contact with the surface of the immersion vibratory compactor during compaction, demonstrates that lower stresses occur in less rigid mixtures as the immersion compactor oscillation has less amplitude.

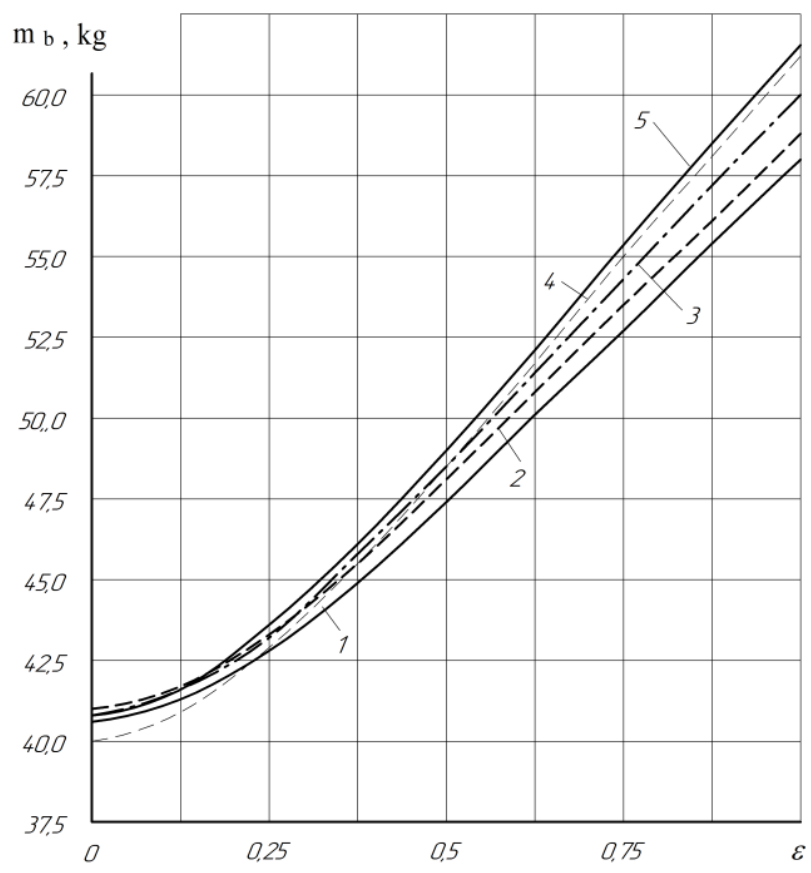

Fig. 5: The variation of the reduced mass of the concrete mixtures $m_{b}$ of different consistence depending on their relative density $\varepsilon: 1-$ at mixture density $\mathrm{S}=6 \mathrm{~s} ; 2-$ at $\mathrm{S}=30 \mathrm{~s} ; 3-$ at $\mathrm{S}=60 \mathrm{~s} ; 4-$ at $\mathrm{S}=90 \mathrm{~s} ; 5-$ at $\mathrm{S}=120 \mathrm{~s}$

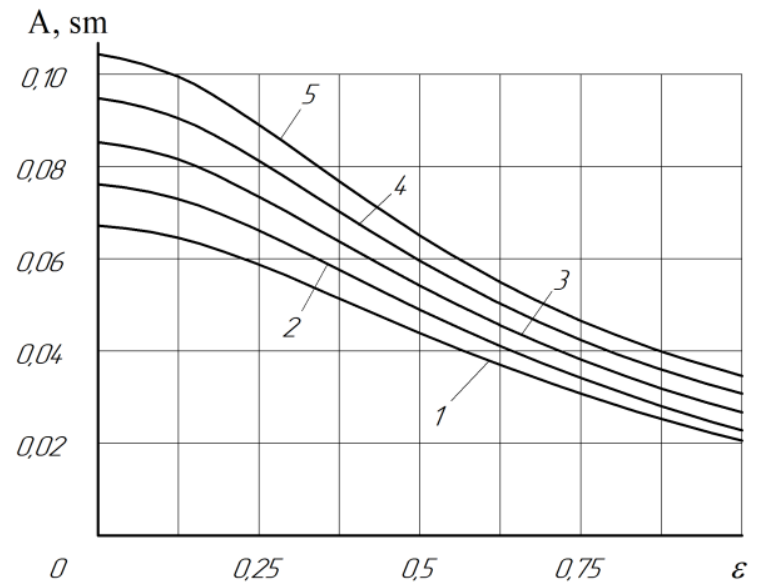

Fig. 6: The variation of the amplitude of the oscillations of the planar immersion vibratory compactor at its interaction with the compacted medium of different consistence depending on the relative density of the concrete mixture: $\varepsilon: 1$ - at mixture density $S=6 \mathrm{~s} ; 2$ - at $S=30 \mathrm{~s} ; 3$ - at $\mathrm{S}=60 \mathrm{~s} ; 4-$ at $\mathrm{S}=90 \mathrm{~s} ; 5-$ at $\mathrm{S}=120 \mathrm{~s}$

Figs. 8 - 9 show the variation of the oscillation amplitudes of the compacted medium and stresses in it depending on the distance to the source of vibration. It is these indices that to a great degree determine the value of the energy pumped into the concrete medium by a planar immersion vibratory compactor, and make it possible to identify the growth of the concrete mixture density in the process of its compaction by the vibratory action. They also enable the determination of the required duration of the vibratory action depending on the concrete mixture consistence and the 
distance along which the waves of resilient-plastic deformations providing the necessary compaction propagate.

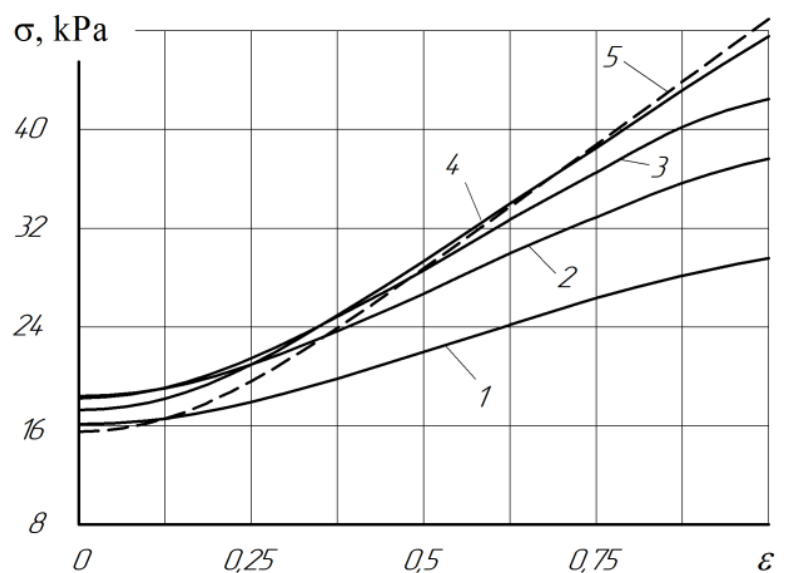

Fig. 7: The variation of the amplitude of the stresses occurring in the concrete medium of different consistence at the place of its contact with the immersion vibratory oscillation compactor depending on the relative density of the concrete mixture: 1 - at the mixture rigidity $S=6 \mathrm{~s} ; 2$ - at $\mathrm{S}=30 \mathrm{~s} ; 3$ - at $\mathrm{S}=60 \mathrm{~s} ; 4-$ at $\mathrm{S}=90 \mathrm{~s} ; 5-$ at $\mathrm{S}=120 \mathrm{~s}$

To provide the concrete mixture effective compaction it is necessary to meet the condition at which the stresses occurring in the compacted layer are to exceed the minimal values of stresses providing the ultimate destruction of the structural links in the compacted medium, i.e.

$\sigma(x) \geq \sigma_{0 i}$,

where $\sigma(x)$ - the amplitude of the stresses occurring in the compacted layer depending on coordinate $x ; \sigma_{0 i}$ - the value of minimal stresses at which the ultimate destruction of the structural links in the compacted medium is provided.

It is at the destruction of the structural links in the compacted medium that its coefficient of internal friction sharply decreases, the mixture passes into thixotropic state, the air is removed out of it, the mobility of the mixture components increases, they reorientate and a more compacted packing is formed.

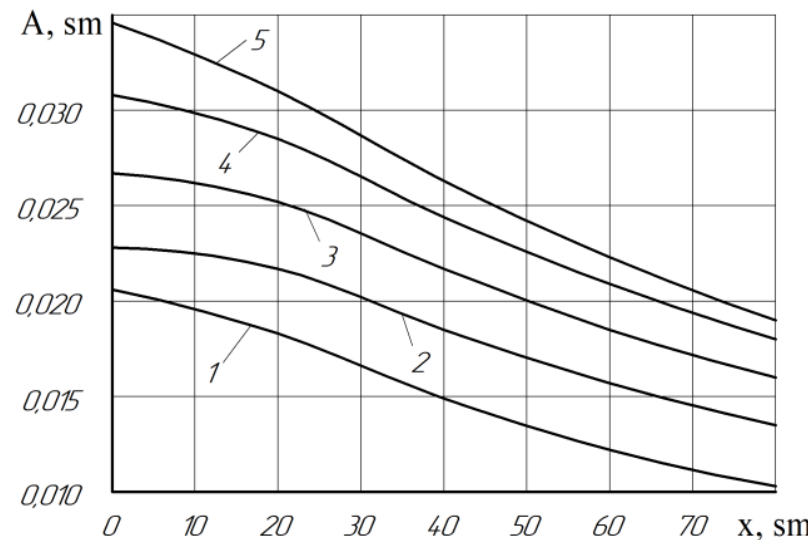

Fig. 8: The variation of the oscillation amplitude of the compacted mixture of different consistence depending on the distance from the source of vibration: 1 - at the mixture rigidity $S=6$ s; 2 - at $S=30$ s; 3 - at $S=60$ s; 4 - at $S=90 \mathrm{~s} ; 5$ - at $S=120 \mathrm{~s}$

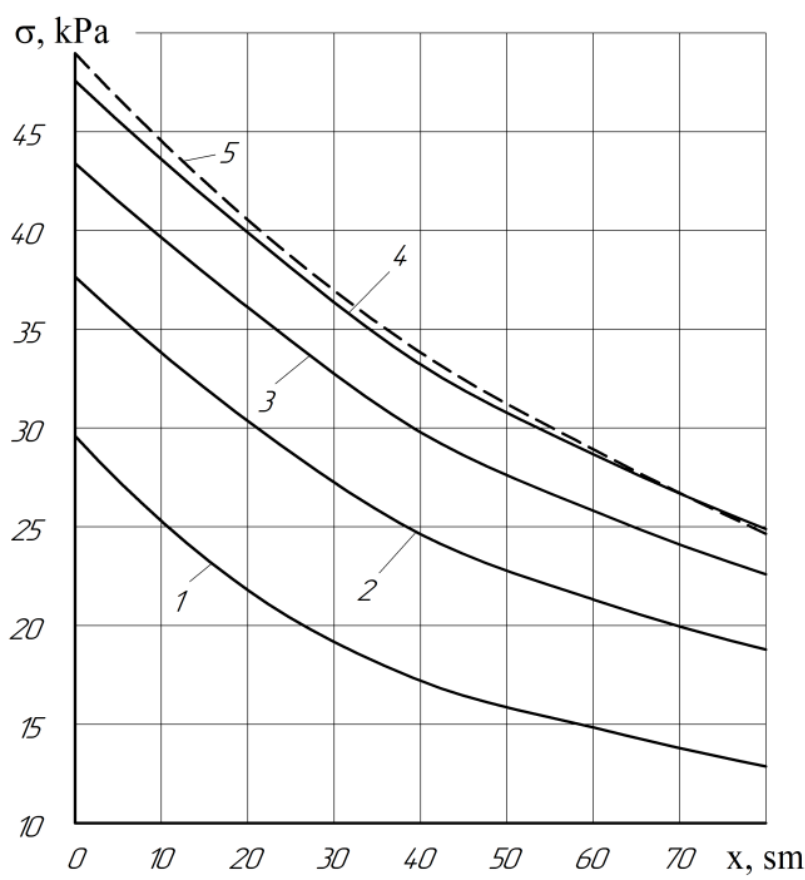

Fig. 9: The variation of the amplitude of the stresses occurring in the compacted mixture of different consistence depending on the distance from the source of vibration: $1-$ at the mixture rigidity $S=6 \mathrm{~s} ; 2-$ at $S=30$ $\mathrm{s} ; 3$ - at $\mathrm{S}=60 \mathrm{~s} ; 4-$ at $\mathrm{S}=90 \mathrm{~s} ; 5-$ at $\mathrm{S}=120 \mathrm{~s}$

\section{Discussion}

When assigning the intensity of the vibratory action it is necessary to take into account the technological condition of inadmissibility of the concrete mixture demixing when the duration of plastic mixtures vibration exceeds (c $S=5-6 \mathrm{~s}$ ) $45 \mathrm{~s}$. The duration of vibration compaction of these mixtures should be 15 to $40 \mathrm{~s}$. For rigid concrete mixtures of the rigidity from $S=30 \mathrm{~s}$ to $S=120 \mathrm{~s}$ the compaction duration should not exceed 120 to $180 \mathrm{~s}$ at their vibration in a form of vertically directed oscillations. When immersion compactors are used, in our opinion, the compaction duration should not exceed 60 to $90 \mathrm{~s}$.

\section{Conclusion}

Based on the analysis of the existing designs and methods of the internal compaction of concrete mixtures, we have proposed a vibration machine in the form of a flat vertical plate, equipped with a vibratory exciter of horizontal oscillations in the upper part. We have created a mathematical model of the dynamic system describing the interaction of the vibration machine with the concrete mixture. We have determined the regularity of the compacted mixture and the vibration machine motion depending on the physical and mathematical characteristics of the compacted medium, the thickness of the compacted layer, the angular frequency of oscillations, the amplitude of the exciting force, and the geometric parameters of the vertical plate interacting with the concrete mixture. The presented dependences provide for substantiation of the rational parameters of the vibration machine and the modes of the vibratory action.

\section{References}

[1] Juradin S, Baloević G \& Harapin A (2014), Impact of Vibrations on the Final Characteristics of Normal and Self-compacting Concrete. Journal of Materials Research, 17(1), pp. 178-185.

[2] Sudarshan NM \& Chandrashekar Rao T (2017), Vibration Impact on Fresh Concrete of Conventional and UHPFRC. International 
Journal of Applied Engineering Research, Vol. 12, 8thedn, pp. 1683-1690.

[3] Koh HB, Yeoh D \& Shahidan S (2017), Effect of re-vibration on the compressive strength and surface hardness of concrete. IOP Conf. Series: Materials Science and Engineering , 271, 012057

[4] Gutierrez J, Ruiz E \& Trochu F (2013), High-frequency vibrations on the compaction of dry fibrous reinforcements. Journal of Advanced Composite Materials, Vol. 22 (1)

[5] Volkov SA \& Evtyukov, S.A. (2012), Construction machinery. $\mathrm{DNK}, \mathrm{SPb}$, Russia

[6] Stacenko AS (2010) Technology of stone works in construction. Vysh. shk., Minsk, Belorussiya.

[7] Gerasimov MD \& Gerasimov DM (2013), Determination of the law of motion, speed and acceleration of the center of mass of the planetary vibration exciter. International Journal of Applied and Fundamental Research, Vol. 12, pp. 8-11.

[8] Chen X, Wu S \& Zhou J, (2013), Experimental study and analytical formulation of mechanical behavior of concrete, Journal of Construction and Buildings Materials, Vol. 47, pp. 662-670.

[9] Banfill PFG, Teixeira MAOM \& Craik RJM (2011), Rheology and vibration of fresh concrete: Predicting the radius of action of poker vibrators from wave propagation. Journal of Cement and Concert Research, Vol. 41, 9thedn, pp. 932-941.

[10] Braun CA, Schumaker M, Rice J \& Borg JP (2015), Comparison of Static and Dynamic Powder Compaction: Experiment and Simulation. Journal of Engineering Materials and Technology, Vol 138, 1 thedn, 011003.

[11] Abdellaoui H \& Echaabi J (2014), Rheological models for modeling the viscoelastic behavior in liquid composite molding processes (LCM) review. Journal of Reinforced Plastics and Composites, Vol. 33, 8thedn, 714.

[12] Sepani H, Polak MA \& Penlidis A (2018), Constitutive Equations and Finite Element Implementation of Isochronous Nonlinear Viscoelastic Behavior. Journal of Engineering Materials and Technology, Vol 140, 4thedn, 041004.

[13] Maslov A.G. \& Salenko Y.S. (2014), Vibrating machines and processes in road construction industry: monograph. PB Cherbatyh, Kremenchuk, Ukraine.

[14] Maslov A.G, Itkin A.F. \& Salenko Y.S. (2014), Vibrating machines for the preparation and compaction of concrete mixes. PB Cherbatyh, Kremenchuk, Ukraine. 